\title{
Herramientas de prevención en las unidades de endoscopia digestiva en época de pandemia
}

\section{Prevention tools in endoscopy suites during the COVID-19 pandemic}

\author{
Robin Prieto, ${ }^{*}$ Gilberto Jaramillo, ${ }^{2}$ Hernán Ballén, ${ }^{3}$ Elder Otero, ${ }^{4}$ Lidsay Delgado, ${ }^{4}$ Juliana Rendón, ${ }^{5}$ Fernando Casas,,${ }^{1}$ Leonardo Fragoso, ${ }^{6}$ \\ María Angélica Rodríguez. ${ }^{7}$
}

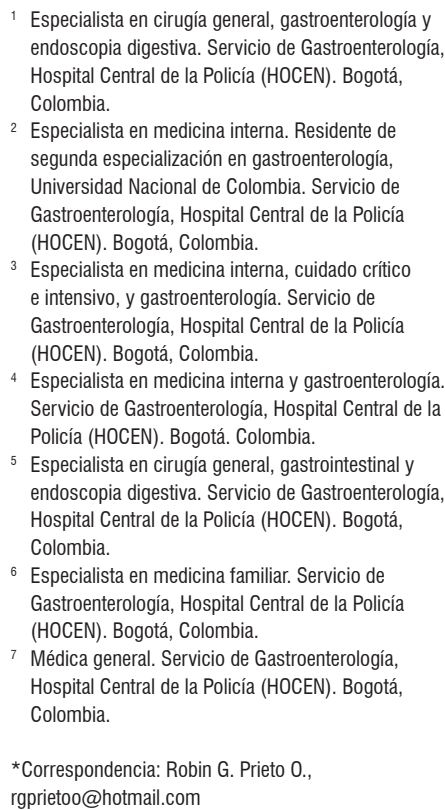

1 Especialista en cirugía general, gastroenterología y endoscopia digestiva. Servicio de Gastroenterología Hospital Central de la Policía (HOCEN). Bogotá, Colombia.

2 Especialista en medicina interna. Residente de segunda especialización en gastroenterología, Universidad Nacional de Colombia. Servicio de Gastroenterología, Hospital Central de la Policía (HOCEN). Bogotá, Colombia.

${ }^{3}$ Especialista en medicina interna, cuidado crítico e intensivo, y gastroenterología. Servicio de Gastroenterología, Hospital Central de la Policía (HOCEN). Bogotá, Colombia.

${ }^{4}$ Especialista en medicina interna y gastroenterología Servicio de Gastroenterología, Hospital Central de la Policía (HOCEN). Bogotá. Colombia.

${ }^{5}$ Especialista en cirugía general, gastrointestinal y endoscopia digestiva. Servicio de Gastroenterología Hospital Central de la Policía (HOCEN). Bogotá, Colombia.

${ }^{6}$ Especialista en medicina familiar. Servicio de Gastroenterología, Hospital Central de la Policía (HOCEN). Bogotá, Colombia.

7 Médica general. Servicio de Gastroenterología, Hospital Central de la Policía (HOCEN). Bogotá Colombia.

*Correspondencia: Robin G. Prieto 0. rgprietoo@hotmail.com

Fecha recibido: $\quad 04 / 04 / 20$ Fecha aceptado: 17/04/20

\begin{abstract}
Resumen
El pasado mes de diciembre surgió en Wuhan, China, el SARS-CoV-2, un nuevo coronavirus productor de la coronavirus disease (COVID-19), que al 15 de abril de 2020 ha infectado a más de 2000000 de personas y ha causado 128833 muertes en el mundo. Por la forma de transmisión del SARS-CoV-2, las salas de endoscopia y los procedimientos endoscópicos son una fuente de fácil diseminación, debido a que cumple con las características de un ambiente contaminado para los pacientes y el personal asistencial que participa en los procedimientos endoscópicos. Por esta razón, es de gran importancia establecer medidas dentro de las unidades de endoscopia para prevenir y disminuir el riesgo de propagación de esta infección, por medio de la creación de un entorno seguro que proteja a los pacientes y al personal de salud. Se realizó una búsqueda en Pubmed con los siguientes términos: ("COVID-19" OR "coronavirus" OR "SARS-Cov-2") y ("gastrointestinal" OR "transmission" OR "intestinal" OR "digestive" OR "endoscopy" OR "esophagogastroduodenoscopy" OR "colonoscopy"). El grupo de gastroenterología de nuestra institución considera que todos los pacientes llevados a procedimientos endoscópicos deben ser catalogados de alto riesgo, por lo cual presenta las recomendaciones que todo el personal asistencial de las unidades de endoscopia debe tener en cuenta para disminuir el riesgo de contagio.
\end{abstract}

\section{Palabras clave}

Endoscopia, gastroenterología, COVID-19, contagio, aerosoles, materia fecal, protección.

\section{Abstract}

Last December, SARS-CoV-2 emerged in Wuhan, China, like a new coronavirus, producing the coronavirus disease (COVID-19), that as of April 15, 2020, it has infected more than 2,000,000 people worldwide and caused 128,833 deaths. Due to the way SARS-CoV-2 is transmitted, endoscopy rooms and endoscopic procedures are an easy source of dissemination, as they fulfill the different characteristics of a contaminated environment for patients and healthcare personnel involved in endoscopic procedures. It is of great importance to establish measures within endoscopy units to prevent and reduce the risk of spreading this infection, creating a safe environment that protects patients and health personnel. A Pubmed search was performed with the following terms: ("COVID-19" OR "coronavirus" OR "SARS-Cov-2") and ("gastrointestinal" OR "transmission" OR "intestinal" OR "digestive" OR "endoscopy "OR" esophagogastroduodenoscopy "OR" colonoscopy "). The gastroenterology team of our institution, considers that all patients brought to endoscopic procedures should be classified as high risk and presents the recommendations that all healthcare staff in endoscopy units, should take into account to reduce the risk of infection.

\section{Keywords}

Endoscopy, gastroenterology, COVID-19, contagion, aerosols, fecal matter, protection. 


\section{INTRODUCCIÓN}

El SARS-CoV-2 es un coronavirus productor de la coronavirus disease (COVID-19), infección potencialmente letal, que tuvo su origen en Wuhan, capital de la provincia de Hubei en China, y fue declarada como pandemia el pasado 11 de marzo por parte de la Organización Mundial de Salud (OMS). Debido al riesgo de transmisión de este virus y al contagio de la enfermedad, la humanidad entera vive una época de transformación en todos los aspectos.

Actualmente, la realización de procedimientos endoscópicos es considerada de alto riesgo, debido a la aerosolización (eructos, vómitos, flatos y presencia de materia fecal), la cual es un factor de riesgo que expone al contagio al personal de salud y a otros pacientes. Es necesario seguir todas las recomendaciones de protección, y definir las pautas adicionales para minimizar el riesgo al que está sometido el personal de salud que participa en la realización de estos procedimientos.

En la literatura, se han definido las características de la transmisión del virus y las medidas de protección para evitar el contagio. Basados en la información médica que cambia día a día y en las observaciones diarias de las rutinas en las salas de procedimientos, presentamos algunas recomendaciones que buscan minimizar el riesgo de transmisión en los servicios de endoscopia.

\section{METODOLOGÍA}

Se realizó una búsqueda en Pubmed con los siguientes términos: ("COVID-19" OR "coronavirus" OR "SARSCov-2") y ("gastrointestinal” OR "transmission" OR "intestinal" OR "digestive" OR "endoscopy" OR "esophagogastroduodenoscopy" OR "colonoscopy"). No hubo restricción de fecha, idioma, ni de ningún otro tipo. Se encontraron 2393 resultados, de los cuales se tomaron los más relevantes.

\section{ANTECEDENTES}

Los coronavirus son virus patógenos para los humanos y para los animales, pertenecen a la familia Coronaviridae y se distinguen 7 especies; poseen una cadena sencilla de ARN, lo que les ha permitido mutar con el paso del tiempo. En noviembre del 2002 en Guangdong, sur de China, surgió el coronavirus del síndrome respiratorio agudo severo (SARS, por sus siglas en inglés) (SARS-CoV), un betacoronavirus que infectó por lo menos a 8000 personas y causó 774 muertes en 37 países. En el año 2012 en Arabia Saudita surgió el coronavirus del síndrome respiratorio del Medio Oriente (MERS, por sus siglas en inglés) (MERS-CoV), responsable de 2494 casos de infección y de 858 muertes (1). El pasado mes de diciembre surgió en Wuhan, China, el SARS-CoV-2, un nuevo coronavirus, productor de COVID-19, que al 2 de abril de 2020 ha causado en el mundo 932501 infectados, 47223 muertes, y 184122 pacientes recuperados. En Colombia, hasta el momento, las cifras son de 1161 infectados, 19 muertos y 55 pacientes recuperados, aunque en todo el mundo se rumora sobre el subregistro (2).

El SARS-CoV-2 está compuesto por 14 residuos de aminoácidos que interactúan con el receptor 2 de la enzima convertidora de angiotensina (ECA), el cual se encuentra en los neumocitos tipo 2, las células miocárdicas, las células del túbulo contorneado proximal del riñón, las células epiteliales de íleon y esófago, y las células uroteliales de vejiga (3). Esto explica sus diversas manifestaciones clínicas, tales como tos, dificultad respiratoria, odinofagia, fatiga/adinamia, fiebre mayor de 38 grados centígrados $\left({ }^{\circ} \mathrm{C}\right)$, náuseas, vómito, diarrea, dolor abdominal, anosmia y ageusia, así como sus diferentes formas de transmisión a través de gotitas respiratorias, ruta fecal-oral y superficies contaminadas (4).

Por la forma de transmisión del SARS-CoV-2, las salas de endoscopia y los procedimientos endoscópicos son una fuente de fácil diseminación debido a que cumplen con las características de un ambiente contaminado para los pacientes y para el personal asistencial que participa en los procedimientos endoscópicos (5). Por lo anterior, es de gran importancia establecer medidas dentro de las unidades de endoscopia para prevenir y disminuir el riesgo de propagación de esta infección, por medio de la creación de un entorno seguro que proteja a los pacientes y al personal de salud.

\section{DEFINICIONES}

Según el Consenso colombiano de atención, diagnóstico y manejo de la infección por SARS-CoV-2, se presentan a continuación las siguientes definiciones (6):

\section{Caso sospechoso}

Persona asintomática (contacto estrecho con un caso confirmado de COVID-19 en los últimos 14 días) o sintomática ( 2 o más de los siguientes síntomas: tos, dificultad respiratoria, odinofagia, fatiga/adinamia, con presencia o no de fiebre mayor o igual a $38^{\circ} \mathrm{C}$, que tuvo contacto con alguna persona que tenga sospecha o confirmación de infección por SARS-CoV-2, o que resida o haya viajado a un área con presencia de casos terciarios de infección en los 14 días previos al inicio de los síntomas, o con imágenes pulmonares con vidrio esmerilado periférico o consolidaciones bilaterales, o persistencia de la sintomatología respiratoria o su empeoramiento al día 8 desde su aparición. 


\section{Caso probable}

Persona con un cuadro clínico sospechoso o asintomático asociado a una prueba rápida positiva o un resultado no concluyente de la reacción en cadena de polimerasa (PCR, por sus siglas en inglés) para la identificación de SARSCoV-2/COVID-19.

\section{Caso confirmado}

Persona asintomática o con un cuadro clínico sospechoso con un resultado positivo en alguna de las pruebas moleculares o genómicas que detectan SARS-CoV-2/COVID 19.

\section{¿QUÉ PROCEDIMIENTOS ENDOSCÓPICOS SE DEBEN REALIZAR?}

Debido a la disponibilidad limitada de recursos (como la escasez de equipo de protección personal [EPP]) combinada con el riesgo de exposición y propagación de la infección para los pacientes y los trabajadores de la salud en el contexto de esta pandemia, las diferentes sociedades y asociaciones mundiales en gastroenterología han recomendado suspender todos los procedimientos endoscópicos programados con fines diagnósticos, y realizar solamente los siguientes procedimientos, que son considerados de urgencia y terapéuticos (7-9):

- Procedimiento para hemorragia digestiva alta en un paciente con inestabilidad hemodinámica.

- Colangitis.

- Extracción de cuerpo extraño.

- Descompresión de vólvulos en obstrucción del tracto digestivo.

- Paliación de obstrucción biliar o luminal del tracto digestivo, endoscopias y colonoscopias cuya importancia diagnóstica sea inaplazable en pacientes con cáncer.

\section{- Gastrostomía.}

Antes de realizar dichos procedimientos, el especialista debe verificar la indicación de estos, los cuales deben ser realizados por el médico disponible más experto. Aún no hay consenso en cuanto al momento indicado para realizar los procedimientos electivos, pero se espera que, con el advenimiento de las diferentes pruebas de tamización y diagnóstico, se pueda estadificar el riesgo de manera más adecuada, para así considerar el reinicio de la realización de estos.

\section{¿QUÉ MEDIDAS DE PREVENCIÓN SE DEBEN TOMAR ANTES Y DURANTE LA REALIZACIÓN DEL PROCEDIMIENTO ENDOSCÓPICO?}

Las diferentes sociedades científicas de gastroenterología han planteado la estratificación del riesgo del paciente con
COVID-19 en bajo y alto, lo que permite determinar el tipo de protección que el personal de salud debe de utilizar (Tablas 1 y 2). Basados en la declaratoria del pasado 31 de marzo del Ministerio de Salud Nacional sobre el inicio de la "fase de mitigación" de la enfermedad (más del $10 \%$ de los casos sin nexo epidemiológico), y sabiendo que más del $60 \%$ de los contagios son ocasionados por personas asintomáticas, nuestro grupo considera que todos los pacientes deben ser catalogados de alto riesgo y recomendamos que todo el personal asistencial de las unidades de endoscopia tome las medidas de protección de alto riesgo (5, 9-11).

Tabla 1. Estratificación del riesgo

\begin{tabular}{|c|c|}
\hline $\begin{array}{l}\text { Pacientes de } \\
\text { bajo riesgo }\end{array}$ & $\begin{array}{l}\text { Sin síntomas } \\
\text { Sin antecedentes de contacto con pacientes COVID-19 } \\
\text { positivos } \\
\text { Que no han viajado a zonas de alto riesgo durante los } \\
14 \text { días anteriores }\end{array}$ \\
\hline $\begin{array}{c}\text { Pacientes de } \\
\text { alto riesgo }\end{array}$ & $\begin{array}{l}\text { Presencia de síntomas sin antecedente de contacto } \\
\text { con alguien que ha dado positivo para COVID-19 } \\
\text { Sin síntomas, pero con: } \\
\text { - Contacto con un paciente que dio positivo para } \\
\text { COVID-19 } \\
\text { - Viaje a área de alto riesgo durante los } 14 \text { días } \\
\text { anteriores } \\
\text { - Por lo menos uno de los síntomas clínicos más uno } \\
\text { de los siguientes: } \\
\text { Contacto con paciente que dio positivo para } \\
\text { COVID-19 } \\
\text { Viaje a área de alto riesgo durante los } 14 \text { días } \\
\text { anteriores }\end{array}$ \\
\hline
\end{tabular}

Modificado de las Recomendaciones de la Sociedad Española de Endoscopia Digestiva (SEED) y de la European Society of Gastrointestinal Endoscopy (ESGE).

Tabla 2. Equipo de protección personal (EPP) de acuerdo con el riesgo

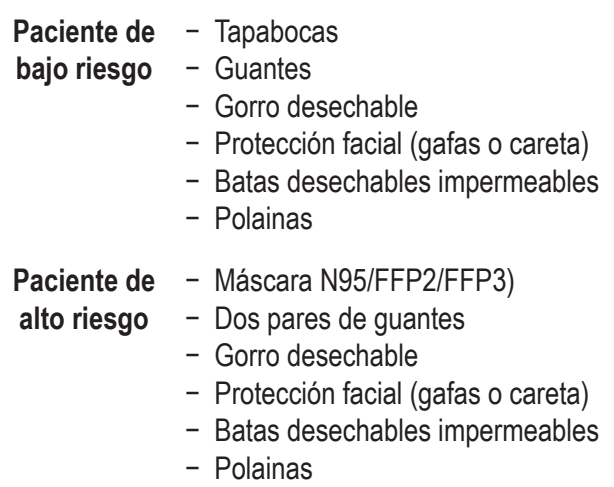

Modificado de las Recomendaciones de la Sociedad Española de Endoscopia Digestiva (SEED) y de la European Society of Gastrointestinal Endoscopy (ESGE). 
De acuerdo con los datos epidemiológicos presentados el 20 de febrero 2020 por la OMS y el Centro Chino para el Control y Prevención de Enfermedades (China CDC) de 2055 trabajadores de la salud infectados durante el brote en la provincia de Hubei, se planteó que los trabajadores de la salud enfrentan una tasa de infección tres veces mayor que la población general (12). Por esta razón, nuestro grupo recomienda la realización de pruebas rápidas serológicas (anticuerpos IgG/IgM) a todo el personal de las unidades de endoscopia digestiva, siguiendo los lineamientos nacionales para el uso de pruebas diagnósticas; esto con el fin de prevenir la propagación del virus e implementar medidas de vigilancia y protocolos de cuarentena.

\section{Medidas preventivas antes de la realización de los procedimientos endoscópicos}

1. Antes de la realización del procedimiento, se debe entrevistar al paciente, para lo cual recomendamos la utilización de un formato en el que se establece el riesgo potencial de infección, y se realiza el seguimiento posterior al procedimiento (Anexo 1).

2. Una vez que el paciente ingrese al servicio, la distancia entre el personal asistencial y el paciente, y entre paciente y paciente, debe ser por lo menos de dos metros $(2 \mathrm{~m})$; de ser posible, se deben utilizar barreras físicas como vidrios.

3. El personal que no está en contacto directo con los pacientes debe usar una máscara quirúrgica.

4. Todo el personal de salud que esté en contacto directo con el paciente debe portar todo el EPP.

5. Verificar que el EPP no haya caducado.

6. Insistir y capacitar a todo el personal sobre el método correcto del lavado de manos y el uso de los diferentes EPP.

7. Los tapabocas son dispositivos sueltos y desechables que deben estar bien adheridos alrededor de la nariz y la boca.

8. Las máscaras N95/FFP2 /FFP3 logran un sello facial muy ajustado, lo que previene la filtración de partículas en el aire $<5 \mu \mathrm{m}$. En el caso de disponibilidad limitada y a pesar de no haber pruebas suficientes para brindar una adecuada confiabilidad en la durabilidad y seguridad, la American Gastroenterological Association (AGA) sugiere el uso extendido o la reutilización de máscaras N95 sobre máscaras quirúrgicas por un período de hasta 8 horas.

9. Es obligatorio lavarse las manos con agua y jabón o usar un desinfectante a base de alcohol antes y después de toda interacción con el paciente, contacto con fuentes potencialmente infecciosas, $y$ antes de ponerse $y$ quitarse el equipo de protección personal, incluidos los guantes.
10. Se debe prohibir estrictamente a los familiares de los pacientes el ingreso a la sala de endoscopia, a menos que el paciente requiera asistencia específica.

11. Todos los pacientes que ingresen a la unidad de endoscopia deben retirarse todos los artículos personales (reloj, anillos, celular, cané, o distintivos), usar una máscara quirúrgica y lavarse las manos.

12. Los baños deben estar completamente separados de los sitios que utilizan los pacientes y el personal de salud por tratarse de lugares potenciales para la propagación del virus. También debe incrementarse la frecuencia de su lavado y desinfección.

13. Debe informarse al paciente sobre el riesgo de adquirir la infección en la sala de endoscopia y, a su vez, consignarlo en el consentimiento informado del procedimiento.

\section{Medidas preventivas durante la realización del procedimiento endoscópico $(5,9-19)$}

Los procedimientos endoscópicos con o sin sedación generan aerosoles; por esta razón, para optimizar el control del riesgo de la infección en la sala de endoscopia, se deben tener en cuenta los siguientes parámetros:

1. La sala de endoscopia debe contar con un sistema de presión negativa o con ventilación adecuada.

2. Se debe restringir el número de personas dentro de la sala de procedimientos (máximo 5) con el fin de disminuir el riesgo de transmisión.

3. En caso de pacientes positivos para COVID-19, se debe marcar la puerta de la unidad de endoscopia para informar que se trata de un paciente aislado y que el acceso a la misma está restringido.

4. Evitar el uso de lidocaína en espray, ya que su administración como anestésico local en la región glótica lleva a que más del $70 \%$ de los pacientes experimenten tos, lo que genera microgotas que pueden transportar el virus y, por su vaporización, la liberación al medio ambiente.

5. Se recomienda que la sedación se lleve a cabo por un especialista en anestesiología.

6. Se recomienda preparar toda la medicación sobre una misma superficie, evitando la manipulación de los carros de medicación. Todo lo que sea necesario para llevar a cabo el procedimiento debe estar disponible en la sala, para evitar la apertura repetida de las puertas después del ingreso inicial del paciente a la sala de procedimientos. En caso de necesitar material del exterior de la sala, se debe asignar un encargado para su transporte hasta la puerta de la sala, donde debe ser recogido por el personal que se encuentra dentro de la sala.

7. Los procedimientos deben realizarse bajo sedación profunda o bajo anestesia general; durante la secuencia 
de intubación, solo deben permanecer dentro de la sala el anestesiólogo y su asistente.

8. De ser posible, se debe evitar la ventilación positiva, el empleo de máscaras laríngeas, de dispositivos tipo fibrobroncoscopio, la intubación en un paciente despierto y el empleo de altos volúmenes en la ventilación mecánica invasiva.

\section{Paso a paso de la colocación del EPP (Figura 1)}

1. Ubique el gorro; recoja y cubra todo el cabello.

2. Realice una higiene adecuada de las manos mediante lavado con jabón, o el uso de una solución a base de alcohol.

3. Vista la bata (preferiblemente con cierre en la espalda, por lo que debe contar con la asistencia de otra persona).

4. Use la máscara facial (N95, FFP2 o FFP3), y verifique que quede ajustada o sellada.
5. Use la protección facial (gafas o careta).

6. Use 2 pares de guantes.

\section{Paso a paso del retiro del EPP (Figura 2)}

El retiro del EPP debe llevarse a cabo con cuidado extremo para evitar la contaminación:

1. Use desinfectante de manos a base de alcohol antes de quitarse los guantes.

2. Retire los guantes.

3. Higienice las manos nuevamente.

4. Use un nuevo par de guantes para evitar la autocontaminación y para continuar el procedimiento de manera segura.

5. Retire la bata. Si usa una bata con cierre posterior, debe ser asistido por otra persona.

6. Retire la máscara facial; evite tocar la parte delantera.

7. Retire las gafas; evite tocar la parte delantera.
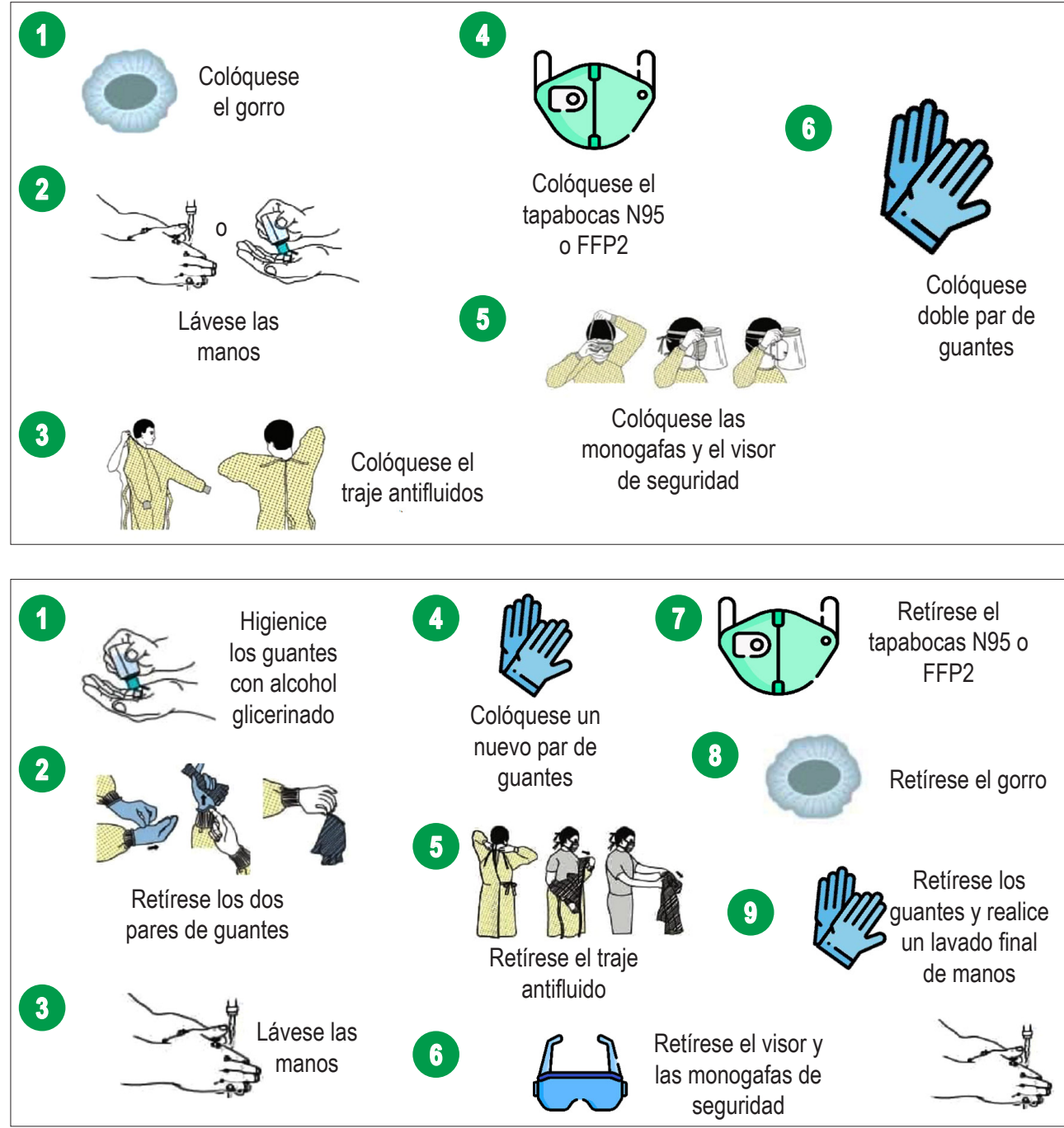

Figura 1. Paso a paso para colocarse el EPP. Modificado de: https:// www.cdc.gov/hai/pdfs/ppe/ppesequence.pdf
Figura 2. Paso a paso para quitarse el EPP. Modificado de: https:// www.cdc.gov/hai/pdfs/ppe/ppesequence.pdf 
8. Retire la máscara (N95 o FFP2).

9. Retire el gorro.

10. Retire el último par de guantes usado.

11. Repita la higiene de las manos.

\section{Reprocesamiento de endoscopios y accesorios}

Cuando se siguen estrictamente todas las pautas actuales de reprocesamiento de alto nivel de desinfección, el riesgo de transmisión de cualquier tipo de virus es extremadamente raro o inexistente. Es obligatorio el uso de productos con características bactericidas, micobactericidas, fungicidas y virucidas contra virus encapsulados y no encapsulados. Se recomienda reforzar capacitaciones al personal en las que se insista en el estricto cumplimiento de las normas de reprocesamiento $(20,21)$.

\section{Desinfección de la sala de endoscopia}

El SARS-CoV-2 es estable en las superficies inanimadas y puede ser una fuente de infección. El desarrollo y la constante auditoría de un detallado plan de limpieza y desinfección de la sala de endoscopia y todo elemento que haya estado en contacto con las manos debe realizarse con una

\section{ANEX0 1}

\section{SERVICIO DE GASTROENTEROLOGÍA HOSPITAL CENTRAL DE LA POLICÍA}

Cuestionario obligatorio a todo paciente que ingrese al servicio de gastroenterología:

Fechadel procedimiento:

Tipodeprocedimientoendoscópico:

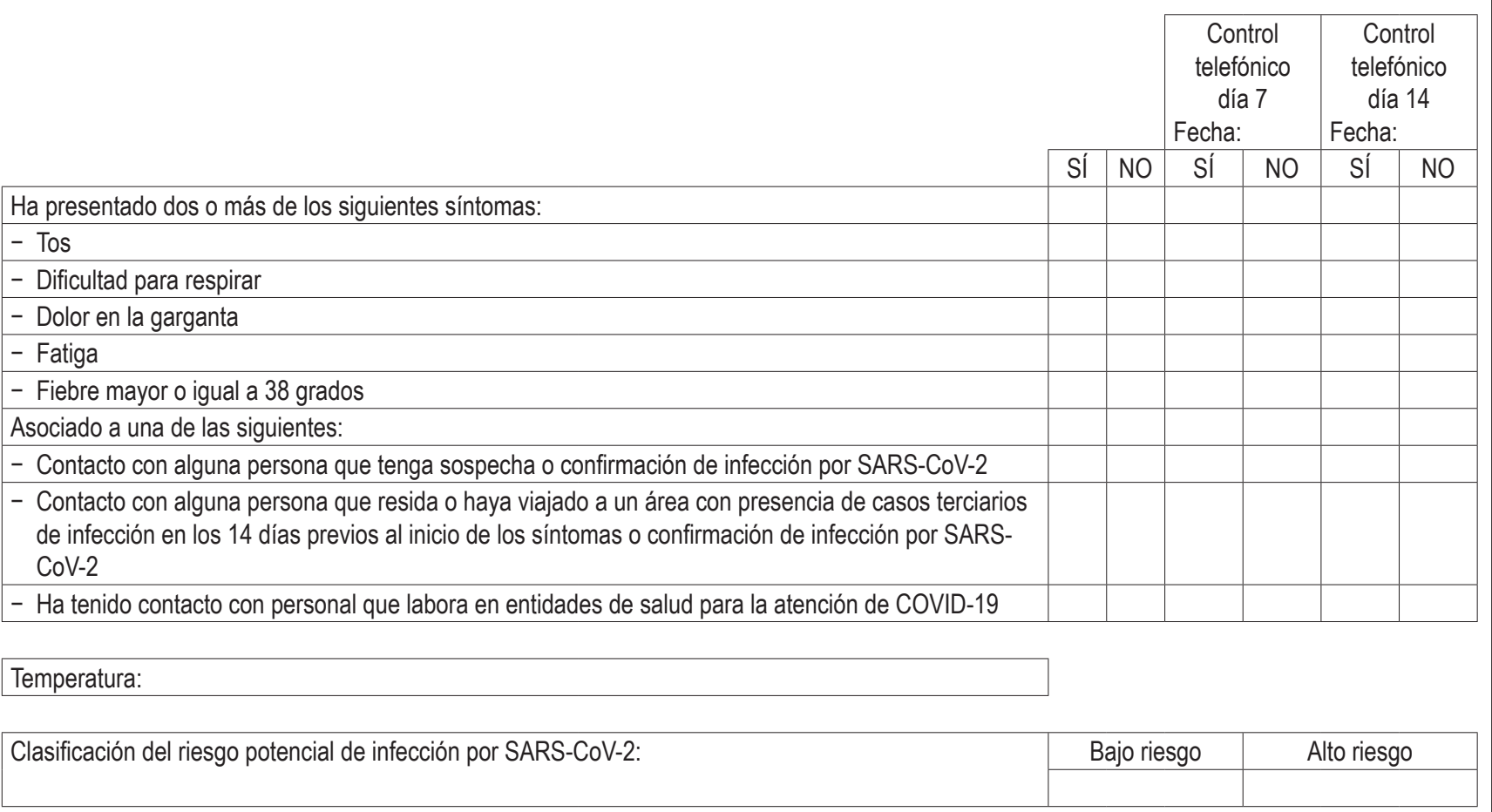

Firma del paciente

Nombre:

Cédula:

Número de teléfono:

Dirección:
Firma del acompañante:

Nombre:

Cédula:

Número de teléfono:

Dirección: 
mezcla de cloro doméstico y agua (dilución 1:100). Este proceso es esencial para disminuir el riesgo de transmisión y debe realizarse al final de cada procedimiento $(22,23)$.

Si el servicio dispone de una sala de procedimientos con presión negativa, se sugiere un tiempo de espera de aproximadamente 30 minutos antes de permitir el ingreso de otro paciente. Si no se dispone de sala de presión negativa, se recomienda un tiempo de recambio de al menos 1 hora, manteniendo la sala desocupada $(22,23)$.

\section{Recomendaciones posprocedimiento $(22,23)$}

1. El retiro y desecho de los elementos de bioseguridad se debe realizar en la misma sala de procedimientos, la cual debe estar separada del resto de las instalaciones de la unidad de endoscopia.

2. Se deben desechar todos los medicamentos e insumos que sobraron o fueron utilizados durante el procedimiento.

3. Se debe contactar a los pacientes al día 7 y 14 para indagar acerca de la aparición de síntomas o del diagnóstico de COVID-19.

\section{CONCLUSIONES}

En el contexto de la pandemia actual, solo se recomienda la realización de procedimientos endoscópicos de urgencias, los cuales deben realizarse preferiblemente bajo sedación o anestesia general con las debidas medidas de precaución para evitar la generación de aerosoles y disminuir el riesgo de contagio.

Se espera que, una vez se tenga la disponibilidad real de las pruebas rápidas y de tamización, se pueda reiniciar la realización de procedimientos endoscópicos electivos, con una mayor seguridad para el personal de salud.

La experiencia vivida por otros países, documentada en la literatura médica, evidencia que el personal de salud tiene tres veces más riesgo que la población general de presentar la infección, por lo que se considera de extrema importancia optimizar y garantizar las medidas de protección del personal de salud en la sala de endoscopia, siguiendo todas las recomendaciones para el correcto uso del EPP.

Todas estas recomendaciones están sujetas al día a día de la evolución de la pandemia.

\section{Conflictos de interés}

Los autores declaramos no tener ningún conflicto de interés.

\section{Financiación}

La financiación se dio con recursos propios de los autores.

\section{REFERENCIAS}

1. Roujian Lu, Xiang Zhao, Juan Li, Peihua Niu, Bo Yang, Honglong $\mathrm{Wu}$, et al. Genomic characterisation and epidemiology of 2019 novel coronavirus: implications for virus origins and receptor binding. Lancet. 2020;395(10224):565574. https://doi.org/10.1016/S0140-6736(20)30251-8

2. WHO [Internet]. Director-General's opening remarks at the media briefing on COVID-19 - 11 March 2020 [acceso 2 de abril de 2020]. Disponible en: https://www.who.int/dg/ speeches/detail/who-director-general-s-opening-remarksat-the-media-briefing-on-covid-19---11-march-2020

3. Zou X, Chen K, Zou J, Han P, Hao J, Han Z. Single-cell RNA-seq data analysis on the receptor ACE2 expression reveals the potential risk of different human organs vulnerable to 2019-nCoV infection. Front Med. 2020. https://doi. org/10.1007/s11684-020-0754-0

4. Guan W, Ni Z, Hu Y, et al. Clinical characteristics of 2019 novel coronavirus infection in China. N Engl J Med. 2020. https://doi.org/10.1056/NEJMoa2002032

5. Repici A, Maselli R, Colombo M, Gabbiadini R, Spadaccini $\mathrm{M}$, Anderloni A, et al. Coronavirus (COVID- 19) outbreak: what the department of endoscopy should know. Gastrointest Endosc. 2020;S0016510720302455. https:// doi.org/10.1016/j.gie.2020.03.019

6. Consenso colombiano de atención, diagnóstico y manejo de la infección por SARS-COV-2/COVID-19 en establecimientos de atención de la salud. Instituto de evaluación tecnológica en salud; 2020.

7. American Society for Gastrointestinal Endoscopy [Internet]. JOINT GI SOCIETY MESSAGE: COVID-19 Clinical Insights for Our Community of Gastroenterologists and Gastroenterology Care Providers [acceso 20 de marzo de 2020]. Disponible en: https://www.asge. org/home/ joint-gi-society-message-covid-19

8. Zhang Y, Zhang X, Liu L, Wang H, Zhao Q. Suggestions for prevention and control in digestive endoscopy during current 2019 - n CoV pneumonia outbreak in Wuhan, Hubei province, China. Endoscopy. 2020;52(4):312-314. https://doi.org/10.1055/a-1128-4313.

9. Sultan S, Lim JK, Altayar O, Davitkov P, Feuerstein JD, Siddique SM, et al. AGA Institute Rapid Recommendations for Gastrointestinal Procedures During the COVID19 Pandemic. Gastroenterology. 2020. https://doi. org/10.1053/j.gastro.2020.03.072.

10. European Society of Gastrointestinal Endoscopy (ESGE) [Internet]. ESGE and ESGENA Position Statement on gastrointestinal endoscopy and the COVID-19 pandemic [acceso 22 de marzo de 2020]. Disponible en: https:// www.esge. com/esge-and-esgena-position-statement-ongastrointestinal-endoscopy-and-the-covid-19-pandemic/

11. Cao Z, Zhang Q Lu X, Pfeiffer D, Jia Z, Song H, Zeng DD. Estimating the effective reproduction number of the 2019nCoV in China. medRxiv. 2020. https://doi.org/10.1101/2 020.01.27.20018952 
12. European Centre for Disease Prevention and Control [Internet]. Guidance for wearing and removing personal protective equipment in healthcare settings for the care of patients with suspected or confirmed COVID-19 [actualizado 28 de febrero de 20202; acceso 27 de abril de 2020]. Disponible en: https://www.ecdc.europa.eu/en/publications-data/guidance-wearing-and-removing-personal-protective-equipment-healthcare-settings

13. WHO [Internet]. Report of the WHO-China joint mission on coronavirus disease 2019 (COVID-19). [acceso 27 de abril de 2020]. Disponible en: https://www.who.int/docs/ default-source/coronaviruse/who-china-joint-missiononcovid 19-final-report.pdf

14. ASGE Quality Assurance in Endoscopy Committee, Calderwood AH, Day LW, Muthusamy VR, Collins J, Hambrick RD 3rd, et al. ASGE guideline for infection control during GI endoscopy. Gastrointest Endosc. 2018;87(5):11671179. https://doi.org/10.1016/j.gie.2017.12.009

15. Beilenhoff U, Biering H, Blum R, Brljak J, Cimbro M, Dumonceau JM, et al. Reprocessing of flexible endoscopes and endoscopic accessories used in gastrointestinal endoscopy: Position Statement of the European Society of Gastrointestinal Endoscopy (ESGE) and European Society of Gastroenterology Nurses and Associates (ESGENA) - Update 2018. Endoscopy. 2018;50(12):1205-1234. https://doi.org/10.1055/a-0759-1629

16. Zhang H, Kang Z, Gong H, Xu D, Wang J, Li Z, et al. The digestive System is a potential route of 2019- nCov infection: a bioinformatics analysis base do singlecell transcriptomes. Microbiology. 2020. https://doi. org/10.1101/2020.01.30.927806

17. Luo S, Zhang X, Xu H. Don't overlook digestive symptoms in patients with 2019 novel coronavirus disease (COVID-
19). Clin Gastroenterol Hepatol. 2020. https://doi. org/10.1016/j.cgh.2020.03.043

18. Chen X, Shang Y, Yao S Liu R, Liu H. Perioperative care provider's considerations in managing patients with the COVID-19 infections. Translational perioperative and Pain Medicine. 2020;7(2):216-224 https://doi. org/10.31480/2330-4871/116

19. Ti LK, Ang LS, Foong TW, Ng BSW. What we do when a COVID-19 patient needs an operation: operating room preparation and guidance. Can J Anaesth. 2020. https://doi. org/10.1007/s12630-020-01617-4

20. Centers for Disease Control and Prevention [Internet]. Coronavirus Disease 2019 (COVID-19) [actualización 23 de abril de 2020]. Disponible en: https://www.cdc.gov/ coronavirus/2019-ncov/infection-control/infection-prevention-control-faq.html

21. Sociedad Española de Endoscopia Digestiva (SEED) [Internet]. Recomendaciones de la SEED: Protección en Unidades de Endoscopia frente al COVID-19 [acceso 23 de marzo de 2020]. Disponible en: https://wseed.es/images/site/guia_clinica/2020/RecomendacionesSEED_ ProteccionUnidadesEndoscopia_Coronavirus.pdf

22. Chen N, Zhou M, Dong X, Qu J, Gong F, Han Y, et al. Epidemiological and clinical characteristics of 99 cases of 2019 novel coronavirus pneumonia in Wuhan, China: a descriptive study. Lancet. 2020;395(10223):507-513. https:// doi.org/10.1016/S0140-6736(20)30211-7.

23. Otero W, Gómez MA, Ángel LA, Ruíz OF, Marulanda H, et al. Procedimientos endoscópicos y pandemia COVID19. Consideraciones básicas. Rev Colomb Gastroenterol. 2020;35(1):65-75. https://doi.org/10.22516/25007440.526 